\title{
Methodical about Methods
}

\author{
Five years ago Nature Cell Biology removed word limits from Methods sections to enhance the detailed description \\ of how experiments are designed, performed and analysed. Here, we revisit our policies that promote full \\ transparency of methodological reporting and the reproducibility of the findings we publish.
}

\begin{abstract}
$\mathrm{f}$ the Results section is the meat of a research paper, the Methods are its backbone. A paper's findings will stand or fall depending on the strength of experimental design and analysis, the quality of reagents, the statistical rigour and the reproducibility of experiments.
\end{abstract} During peer review, referees assess these key elements, together with the presented data, in order to determine whether conclusions are sufficiently supported, to identify experimental weaknesses and gaps in reasoning, and to propose how best to address them. Therefore, detailed reporting of methodology is instrumental to improving a paper before it reaches the public domain.

With the exception of studies reporting game-changing technical advances, after publication the Methods section is less likely to be read in depth by most readers. Dry, terse and littered with details on reagents, model systems, and experimental and statistical analyses, it is not typically the part of a manuscript that will excite the general reader and set a broad audience abuzz. However, it is the essential part that will solidify the reported findings and propel the field forward. Given sufficient detail, the validation and direct follow up of a paper's experiments can be relatively straightforward. But omitting a few key details can cause researchers to struggle to replicate even basic experiments, and in the worst cases can lead to questions about data validity and conflicting reports. Anecdotes abound of researchers following a trail of papers cited from one Methods section to the next, only to discover that the referenced methodology was not actually described in the purportedly original publication.

Recognising the need for detail in this core part of research manuscripts and to provide sufficient space for the full description of methodology, reagents and analysis, Nature Cell Biology lifted restrictions on the length of the Methods in 2013 (https://go.nature. com/2JFBJLM). Since then our authors have made good use of the extra space, with the average Nature Cell Biology paper published in the first trimester of 2018 having doubled the Methods' length to 2,700 words, compared to an average $\sim 1,370$ words in papers published in the three months prior to removing length limits. In 2013, we also introduced the Reporting Checklist of methodological and statistical information. As this checklist was not published, editors worked diligently with authors and reviewers to ensure that all essential information was included in the Methods for publication. Last year we enhanced these efforts by revamping the original checklist into the Reporting Summary (https://go.nature.com/2GW4EgE and https://go.nature.com/2qscaFZ). This updated document is now published with the manuscript, as it aims to directly complement the Methods by listing details on experimental design, statistical analysis and key reagents in a structured manner. Thus, it also includes discrete sections that capture information on approaches such as flow cytometry, chromatin immunoprecipitation sequencing and magnetic resonance imaging, with more techniques to be included in the future. Accompanying the Reporting Summary is the Editorial Policy Checklist, which is not published but rather guides authors through the policies they need to follow and information they should include in the Methods, depending on the type of research conducted.

Together, the Methods section and Reporting Summary permit authors to describe reagents and how experiments were performed and analysed in great depth. Our long-standing policies requiring detailed information on reagents such as antibodies and mammalian cell lines, for which source and validation is key (https://go.nature. com/2HvVMeW and https://go.nature. $\mathrm{com} / 2 \mathrm{qrXAO1}$ ), remain unchanged. However, authors are now prompted to provide these details in a structured manner through the Reporting Summary. Similarly, information on model organisms and experiments involving human participants or samples are requested in this document, with authors asked to declare protocol approval by relevant boards and institutions, compliance with ethical guidelines and informed consent by human participants in more detail in the Methods.

We also continue to strongly encourage the deposition of step-by-step protocols in the Protocol Exchange (https://go.nature. com/2qrgJiR), an open-access resource maintained by Nature Protocols. Deposited protocols can be cited in the Methods and we mandate this for papers reporting new or substantially updated methodology, or when a technical aspect is deemed central to the manuscript conclusions.

This journal has long championed the sharing of data by mandating deposition of specific data types including, among others, array, sequencing, macromolecular and proteomic data to specific repositories (see https://go.nature.com/2EFF1Ld), and by recommending the provision of source data (https://go.nature.com/2JFBJLM). Since 2016, our Methods sections have included a mandatory 'Data Availability' space where authors report the availability of the data supporting the findings of the manuscript, through accession numbers and links to repositories, source data within the manuscript, or through the authors themselves (https:// go.nature.com/2qpuUVV). Studies presenting custom-made computational code that is central to the paper's conclusions must include sufficient detail in the Methods for readers to follow the conclusions, and we consider it best practice to release the code to allow others to repeat the results independently. How the code can be accessed is noted in a separate 'Code Availability' section of the Methods (see also http://go.nature.com/2D2l80d).

Finally, we continue to place great weight on the reporting of statistical descriptions. The Reporting Summary and Editorial Policy Checklist prompt authors to describe study design and statistical parameters in the relevant sections of the manuscript. Detailed statistical descriptions belong in the figure legend specific to each experiment, to permit readers to interpret experiments accurately. However, the Methods include a mandatory 'Statistics and Reproducibility' section which provides general information on statistical analyses and reproducibility of the experiments presented in the paper.

With the combination of Reporting Summary and Methods with discrete sections, and with close editorial guidance to authors, we aim to provide improved and more consistent methodological descriptions. Our commitment to enhancing the quality and transparency of reporting means that our policies keep evolving, and as always the feedback of our authors, reviewers and readers is welcome at ncb@nature.com.

Published online: 25 April 2018

https://doi.org/10.1038/s41556-018-0103-6 